\title{
Clinical Validity of the NIMHANS Sentence Completion Test for Children and Adolescents
}

\author{
Dhanya V.S. ${ }^{1}$ \\ Snigdhasree Bhattacharya ${ }^{2}$ \\ Uma Hirisave ${ }^{3}$ \\ L.N. Suman ${ }^{4}$ \\ ${ }^{1}$ Clinical Psychologist, Government Medical College, Thissur, Kerala. \\ ${ }^{2}$ PhD Scholar, Department of Clinical Psychology, NIMHANS, Bangalore. \\ 3,4 Professor, Department of Clinical Psychology, NIMHANS, Bangalore. \\ E-mail-snigdhab1@yahoo.com
}

\begin{abstract}
The present study, aims to examine the adjustment and social problem solving skills in children with emotional and behavioral problems using the NIMHANS Sentence Completion test for Children and Adolescents (NSCT). NIMHANS Sentence Completion test for Children and Adolescents is a standardized semi projective test for children and adolescents in the Indian setting. The test has 75 items, which are easy to follow and culturally relevant. The test has been developed following the steps prescribed for construction of a psychological test and the test has sound psychometric properties.In the current study the sample consisted of 30 children with a psychiatric diagnosis of Behavioral and Emotional Disorder (F 91-98); Depressive Disorder (F 32 and F 33); or Dissociative Disorder ( $(F 44)$ on Axis I of the Multi-axial Classification System of ICD-10. The sample also consisted of 30 normal children selected from English medium schools. The results indicate that NSCT correlated significant with 'Psychosocial Stress' as assessed by the DPCL ( $r=0.35)$ which was significant at the .05 level). That is higher the stress, poorer the adjustment as measured by the NSCT. Apart from this comprehensive qualitative analysis also indicates significant difference in the way clinical and normal children respond to the items. The results indicate good clinical validity of the scale. The authors have also enclosed the NSCT in the article giving readers an opportunity to use it for clinical or research based application of the test.
\end{abstract}

Key words: Projective testing in children, NIMHANS Sentence Completion Test in Children and Adolescent, Assessing adjustment in children.

\section{INTRODUCTION}

NIMHANS Sentence Completion test for Children and Adolescents is a standardized semi projective test for children and adolescents in the Indian setting. The test has 75 items, which are easy to follow and culturally relevant. The test has been developed following the steps prescribed for construction of a psychological test and the 
test has sound psychometric properties [1]. The Sentence completion test is based on the theory which deals with the importance of adjustment in several areas pertinent to child's emotional and development. The test was developed to assess the child's perceptions, attitude and interactions to arrive at a comprehensive understanding of the child's adjustment. The test covers 3 main domains such as home, school/peers, self and neutral items. The sentence stems representing different sub areas were ordered in a serial manner so that items in any specific area are not clustered together. Both positive and negative stems were included. The Scoring system suggested by Sack [2] was adopted wherein the score for each area had to be 0,1 or 2 . 0 indicates good adjustment, 1 indicates mild problem in adjustment, and 2 indicates poor adjustment. The aim of the current study was to examine the adjustment and social problem solving skills in children with emotional and behavioral problems.

\section{METHODOLOGY}

The sample consisted of 30 children with a psychiatric diagnosis of Behavioral and Emotional Disorder (F 91-98); Depressive Disorder (F 32 and F 33); or Dissociative Disorder ( $(F$ 44) on Axis I of the Multi-axial Classification System of ICD-10 [3]. Care was taken to include only new cases registered in the CAMH Unit of NIMHANS [4]. The sample also consisted of 30 normal children selected from English medium schools. They were screened on the Children's Behavior Questionnaire [5] to rule out children with psychological problems. The age range of the sample was 8 to 12 years and both boys and girls were included in both the groups. The profile of emotional and behavioral problems was obtained using the Developmental Psychopathology Checklist [6]. The Developmental psychopathology Checklist has been standardized on 221Indian children below 16 years of age and has been found to have sound psychometric properties. Adjustment was assessed using the NIMHANS Sentence Completion test for Children and Adolescents [1] and Social Problem Solving was assessed using Group Social Problem Solving Assessment [7]. Written informed consent was taken from parents and teachers and assent for the study was taken from the children.

\section{RESULTS}

Results of the study as given in Table 1 indicate that there was a significant difference between the two groups on overall adjustment as measured by the NSCT.

Table 1 - Difference between groups on overall adjustment

\begin{tabular}{|llllllll|}
\hline Group & & N & Mean & SD & T & & \\
\hline SCT & Clinical & 30 & 41.63 & 11.89 & t value & df & $p$ Value \\
\hline $\begin{array}{l}\text { Total } \\
\text { Score\# }\end{array}$ & Normal & 30 & 31.77 & 10.44 & 3.80 & 54 & $\begin{array}{l}0.001^{*} \\
\text { Significant }\end{array}$ \\
\hline
\end{tabular}

\#In the study, each item has been rated on the three point scale ranging from 0 to 2; thus the maximum possible score is 140 ( 70 items multiplied by two; neutral items are not scored) 
Analysis of the responses in terms of sub-areas revealed significant differences between the two groups in five areas. These were: Adjustment to school; Emotional Functioning; Coping with Emotions; Parental attitude towards the child; and Guilt Feelings. There were no significant differences between the two groups in the other ten sub- areas. Details of significant differences are given in Table 2.

Table 2 - Differences between groups on the sub-scales

\begin{tabular}{|c|c|c|c|c|c|}
\hline Area & Group & $\mathbf{N}$ & Mean & SD & $\mathbf{T}$ \\
\hline $\begin{array}{l}\text { Adjustment to } \\
\text { School }\end{array}$ & $\begin{array}{l}\text { Clinical } \\
\text { Normal }\end{array}$ & $\begin{array}{l}30 \\
30\end{array}$ & $\begin{array}{l}0.73 \\
0.73\end{array}$ & 3.7 & $0.001^{*}$ \\
\hline $\begin{array}{l}\text { Emotional } \\
\text { Functioning }\end{array}$ & $\begin{array}{l}\text { Clinical } \\
\text { Normal }\end{array}$ & $\begin{array}{l}30 \\
30\end{array}$ & $\begin{array}{l}0.52 \\
0.50\end{array}$ & 2.8 & $0.007^{*}$ \\
\hline $\begin{array}{l}\text { Coping with } \\
\text { Emotions }\end{array}$ & $\begin{array}{l}\text { Clinical } \\
\text { Normal }\end{array}$ & $\begin{array}{l}30 \\
30\end{array}$ & $\begin{array}{l}0.69 \\
0.50\end{array}$ & 2.3 & $0.022 * *$ \\
\hline $\begin{array}{l}\text { Parental } \\
\text { Attitude } \\
\text { towards Child }\end{array}$ & $\begin{array}{l}\text { Clinical } \\
\text { Normal }\end{array}$ & $\begin{array}{l}30 \\
30\end{array}$ & $\begin{array}{l}0.52 \\
0.50\end{array}$ & 3.5 & $0.001 *$ \\
\hline $\begin{array}{l}\text { Guilt } \\
\text { Feelings }\end{array}$ & $\begin{array}{l}\text { Clinical } \\
\text { Normal }\end{array}$ & $\begin{array}{l}30 \\
30\end{array}$ & $\begin{array}{l}0.80 \\
0.47\end{array}$ & 3.9 & $0.000 *$ \\
\hline
\end{tabular}

*Significant at 0.05 level; $* *$ significant at .001 level

\section{DISCUSSION}

The study aimed to assess adjustment in children with emotional and behavioral problems and normal children. The NIMHANS Sentence Completion Test for children and adolescents (NSCT) was administered to understand more about the areas of adjustment among the two groups. When the results of the clinical and normal group were compared on NSCT (Table 1), it was found that there is significant difference between the two groups on overall adjustment. Children in the clinical group reported much more difficulties in overall adjustment when compared to the normal group.

Further analysis revealed that there was significant difference noted in adjustment in sub domains of school, emotions, coping with emotions, attitude of parent towards the child and guilt feelings (Table 2). Children in the clinical group were found to have significantly more difficulties in these areas when compared to the children from the normal group. These findings highlight the major areas of difficulties and adjustment seen in clinic children. The findings provide an insight in the clinical group's areas of difficulties and can guide the process of intervention successfully.

However in the sub domains of adjustment such as relationship with father, mother, siblings, friends, teachers, relationship between father and mother and in the areas own abilities and goals there is no significant difference in the two groups. This is indicative that children in clinical setting adjusted well in these areas, it can be attributed to their resilience which is in turn strength in the process of intervention.

In the School area, responses of clinic children revealed more difficulties in school such as fear of school itself and fear of examinations. They also indicated that most of their friends were from their home networks rather than school 
mates. On comparing the responses of the two groups on the domain of Emotional Functioning, it was found that children in the clinical group reported more negative emotions such as anxiety and fears. The two groups also differed in the way in which they coped with emotions. Children in the clinical group had poorer coping skills compared to normal children. Further, children in the clinical group perceived their parents as having less favorable attitude towards them, which in turn contributed to their adjustment difficulties. Children in the clinical group also had significantly high Guilt Feelings. Their guilt was mainly related to being a burden on their parents due to their illness/difficulties. Children in the normal group had positive attitudes towards school and teachers and a good self-concept.

It was found that most of the children with a primary diagnosis of conduct disorder reported that they were not fearful of anything and many of them expressed their sadness as anger in multiple contexts. Thus it is important to address these internalizing symptoms even though children may get a diagnosis of conduct disorder. Fear of darkness and ghosts were commonly expressed by clinical normal groups. The clinical group children showed difficulty in appropriately expressing their emotions. Many showed a tendency to express anger as sadness and vice versa. They also reported disproportionately high emotional responses to different contexts.

On exploring the child's perception of parental attitude towards them, many children in the clinical group reported their parents view them as different from others, though not as inferior. Similar responses were seldom reported by children from normal sample. General trend observed in the clinical sample is use of more negatively loaded words such as "I hate my father, he is my enemy", where as in the normal sample children used more subtle expression of dislike such as "disappointed with father at times". When asked about the teachers, some of the clinical sample complained teachers being very partial and not understanding; whereas children of the normal sample expressed their dislike to specific events like "punishing when I don't do my homework". Children from normal sample expressed dislike to specific situations unlike clinical sample that attributed negative qualities as more global and stable. Ironically most of the children who reported strong dislike towards teachers or difficulties with school were not the ones who were having co morbid learning problems.

Most of the children gave favorable responses about mother, though some of them tend to give more impersonal responses like for the same stem sentence- my mother is "cooking", "in office" etc. This was similar in case of fathers too. The NSCT also correlated significant with 'Psychosocial Stress' as assessed by the DPCL ( $r=0.35$ which was significant at the .05 level). That is higher the stress, poorer the adjustment as measured by the NSCT. Thus, the results obtained in the study revealed that the test could significantly discriminate between the two groups. This indicates good clinical validity and usefulness. With regard to the process of administration, there was a general tendency in both the groups to give more socially appropriate statements for the initial items. They tend to become more open after few items. Most of the assessment was manifested either by giving neutral responses or by increased reaction time. This is similar to interpretation of increase reaction time as a sign of disturbance in other projective tests [8].

Normal group children were more spontaneous in responses and needed fewer explanations from the examiner during assessment. Clinical group children needed more to get comfortable with the testing situation. The responses of sample were similar to the responses of the original sample in which the tool was standardized. The children from the original sample who were well adjusted had similar responses as that of the normal children in the current study. Thus the qualitative and quantitative 
analysis of current data with regard to adjustment indicated that most of children from the clinical sample had more psycho social stressors in their life, which would have contributed to their difficulties in adjustment in various domains. The responses of clinical group and clinical group were different both content wise and the way in which they approached the task. Therefore to conclude with, the study has made a valuable contribution in establishing the clinical validity of the NIMHANS Sentence Completion Test for Children and Adolescents. The study highlights that semi-projective tests can provide rich material regarding children's difficulties and adjustments. It has also given an insight and information about the nature and extent of the difficulties reported among the clinical group of children. The responses can be used as a spring board to carry out in-depth interviews to plan psychological interventions. The test can also be used as a pre and post measure of adjustment in intervention studies or in routine clinical practice.

\section{REFERENCES}

1. Bhattacharya S, Hirisave U, Suman LN. Development of the NIMHANS Sentence Completion Test for Children and Adolescents. Indian Journal of Mental Health $2015 ; 2(1)$ : 63-8.

2. Sacks JM, Levy S. The Sentence Completion Test. In LE Abt and L Bellak (Eds.) Projective Psychology (pp. 357-402). New York:Knopf;1950.

3. World Health Organization (WHO). The ICD-10: Classification of Mental and Behavioral Disorders; Clinical descriptions and guidelines. Geneva, A.I.T.B.S Publishers ; 1992.

4. Dhanya VS. Adjustment and social problem solving skills in children with emotional and behavioral problems. Unpublished M.Phil Dissertation submitted to NIMHANS (Deemed University): Bangalore ; 2009.

5. Rutter M. A Children's Behavior Questionnaire for completion by teachers: Preliminary findings. J Child Psychol Psychiatry 1967;8:1-12.

6. Kapur, M, Barnabas I, Reddy MV, Rozario J, Uma H. Development of a checklist for assessment of childhood psychopathology in the Indian setting. Indian Journal of Clinical Psychology 1994;21:40-2.

7. King D. Broad-based support pushes health education beyond what the coach does between seasons. ASCD Curriculum Update 1986;1:1-12.

8. Klopfer B, Ainsworth MD, Klopfer WG and Holt RR. Developments in the Rorschach technique, Vol. 1 Harcourt, Brace and World, Inc: New York; 1954.

Acknowledgements - Nil

Conflict of Interest - Nil

Funding - Nil. 


\section{APPENDIX \\ NIMHANS Sentence Completion Test \\ For \\ Children and Adolescents (NSCA) Final Version \\ Department of Clinical Psychology, NIMHANS, Bangalore}

Instructions: You will find below a number of incomplete sentences. For example: "The colour of the leaf is ......................." Write/tell quickly in the blank space the first idea that comes to your mind to complete each sentence. Quickness is very important, please answer rapidly.

1. My father is

2. My friend and I

3. My teachers are

4. My mother and I

5. At school, I feel

6. I have the ability to

7. My brother is

8. I am afraid of

9. My mother and my father

10. I like to

11. When I am afraid, I

12. My parents think I am

13. My ambition is

14. In my family

15. My biggest mistake was

16. I like my father because

17. Most of my friends

18. My teachers and I

19. I like my mother because

20. I like going to school, but.

21. When things go wrong I.

22. My sister is

23. I feel happy when

24. My father treats my mother

25. My favorite eatable is

26. When I am happy, I

27. My parents feel happy when I

28. I want to become

29. My grand father

30. I wish I had not

31. My father and I

32. A real friend is

33. I feel my teachers

34. My mother

35. I find class tests

36. In order to achieve something, I can.

37. My brother and I

38. I feel anxious when 
39. I wish my father and mother

40. On Sundays I like to

41. When I am anxious, I

42. My parents scold me when

43. I will be happy if I can

44. My family treats me

45. I feel guilty about

46. I wish my father

47. My best friend

48. I like my teachers but

49. I dislike my mother because

50. The subject I dislike most

51. Others say I am capable of

52. My sister and I

53. I feel angry when

54. My mother treats my father

55. I am interested in

56. When I am angry, I

57. My parents get angry when I

58. I always wanted to be

59. The person I like most in my family

60 . I wish I could forget

61. I dislike my father because

62. I expect my friends to

63. Most teachers are

64. I wish my mother

65. I like my brother but sometimes

66. I feel sad when

67. My grandmother

68. When I am sad, I

69. My parents beat me when

70. I wish my family

71. The worse thing I ever did was

72. Most families I know are

73. I like my sister but sometimes

74. I like my family but sometimes

75. My favourite game is

For scoring and further details please contact Snigdhasree Bhattacharya (author no 2) on snigdhab1@yahoo.com 